Article

\title{
Ergostane-Type Sterols from King Trumpet Mushroom (Pleurotus eryngii) and Their Inhibitory Effects on Aromatase
}

\author{
Takashi Kikuchi ${ }^{1}$, Naoki Motoyashiki ${ }^{1}$, Takeshi Yamada ${ }^{1}{ }^{(\mathbb{C})}$, Kanae Shibatani $^{2}$, \\ Kiyofumi Ninomiya ${ }^{2}$, Toshio Morikawa ${ }^{2, *}$ (i) and Reiko Tanaka ${ }^{1, *}$ \\ 1 Faculty of Pharmaceutical Sciences, Osaka University of Pharmaceutical Sciences, 4-20-1 Nasahara, \\ Takatsuki, Osaka 569-1094, Japan; t.kikuchi@gly.oups.ac.jp (T.K.); mottchan050321@gmail.com (N.M.); \\ yamada@gly.oups.ac.jp (T.Y.) \\ 2 Pharmaceutical Research and Technology Institute, Kindai University, 3-4-1 Kowakae, Higashi-osaka, \\ Osaka 577-8502, Japan; 1633420001c@kindai.ac.jp (K.S.); ninomiya@phar.kindai.ac.jp (K.N.) \\ * Correspondence: morikawa@kindai.ac.jp (T.M.); tanakar@gly.oups.ac.jp (R.T.); Tel.: +81-6-4307-4306 (T.M.); \\ +81-72-690-1084 (R.T.); Fax: +81-6-6729-3577 (T.M.); +81-72-690-1084 (R.T.)
}

Received: 18 October 2017; Accepted: 16 November 2017; Published: 21 November 2017

\begin{abstract}
Two new ergostane-type sterols; (22E)-5 $\alpha, 6 \alpha$-epoxyergosta-8,14,22-triene-3 $3,7 \beta$-diol (1) and $5 \alpha, 6 \alpha$-epoxyergost-8(14)-ene- $3 \beta, 7 \alpha$-diol (2) were isolated from the fruiting bodies of king trumpet mushroom (Pleurotus eryngii), along with eight known compounds (3-10). All isolated compounds were evaluated for their inhibitory effects on aromatase. Among them, $\mathbf{4}$ and $\mathbf{6}$ exhibited comparable aromatase inhibitory activities to aminoglutethimide.
\end{abstract}

Keywords: Pleurotus eryngii; sterol; ergostane; aromatase inhibitor

\section{Introduction}

Estrogen is responsible for breast cancer growth. The target genes of an estrogen receptor are in control of cancer cell development in estrogen-dependent breast tumors. Binding of estrogen receptor to estrogen triggers transcription of its target genes [1]. Aromatase is the rate-limiting enzyme in estrogen biosynthesis [1]. This enzyme converts androgens (testosterone and androtestosterone) into estrogens (estradiol and estrone, respectively) [2]. Aromatase inhibitors (AIs) are adjuvant in hormone treatments commonly prescribed for breast cancers that are hormone receptor-positive in the early stage [3]. However, the currently used AIs have several side effects of menopausal symptoms such as hot flashes, vaginal dryness, sexual dysfunction, musculoskeletal symptoms, osteoporosis, bone fracture, fatigue, mood disturbance, nausea, and vomiting [3]. Therefore, natural compounds obtained from safe food resources might be useful in the search for promoter-specific AIs with few side effects [4].

Pleurotus eryngii (Japanese name: eringi, English name: oyster mushroom or king trumpet) is an edible mushroom. P. eryngii is native to North Africa, Asia, and Europe [5], and also grown commercially in Japan, China, and the US [6]. Previous studies demonstrated the inhibitory effects on human neutrophil elastase (HNE) [7], antioxidant and antimutagenic activities [8], and inhibitory effects on allergic mediators [9] of P. eryngii extracts. P. eryngii contains amino acids, vitamins, and dietary fiber [10]. It also includes polysaccharides [11,12], pleurone [7], ergostane-type sterols [13], and eryngiolide A [14]. These chemical constituents exhibit biological activities such as the antioxidant [11] and antitumor activities [12] of a polysaccharide, HNE-inhibitory effects of pleurone [7], and cytotoxicity against human cancer cell lines of eryngiolide A [14]. We recently reported eringiacetal A, which is an ergostane-type sterol with a cage-shaped 
structure [15], and a 9,11-seco-ergostane and five ergostane-type sterols [16] from the fruiting bodies of $P$. eryngii. In a continuing study, we isolated 10 ergostane-type sterols, and elucidated the structures of two new compounds; (22E)- $5 \alpha, 6 \alpha$-epoxyergosta-8,14,22-triene-3 $\beta, 7 \beta$-diol (1), and $5 \alpha, 6 \alpha$-epoxyergost-8(14)-ene-3 $\beta, 7 \alpha$-diol (2). In addition, the isolated constituents were evaluated for inhibitory activities on aromatase.

\section{Results}

\subsection{Isolation and Structure Elucidation}

(22E)-Ergosta-7,22-dien-3ß-ol (3) [17], (22E)-ergosta-5,7,22-trien-3ß-ol (4) [18], (22E)-19-norergosta-5, 7,9,22-tetraen-3 $\beta$-ol (5) [19], ergosterol peroxide (9) [18], and 9,11-dehydroergosterol peroxide (10) [20] were isolated from sample 1 , and Compounds 1, 2, (22E)-6 $\beta$-methoxyergosta-7,22-diene-3 $\beta, 5 \alpha$-diol

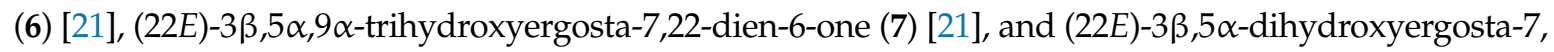
22-dien-6-one (8) [22] were obtained from sample 2 (Figure 1). Of these, $\mathbf{1}$ and $\mathbf{2}$ were new compounds.

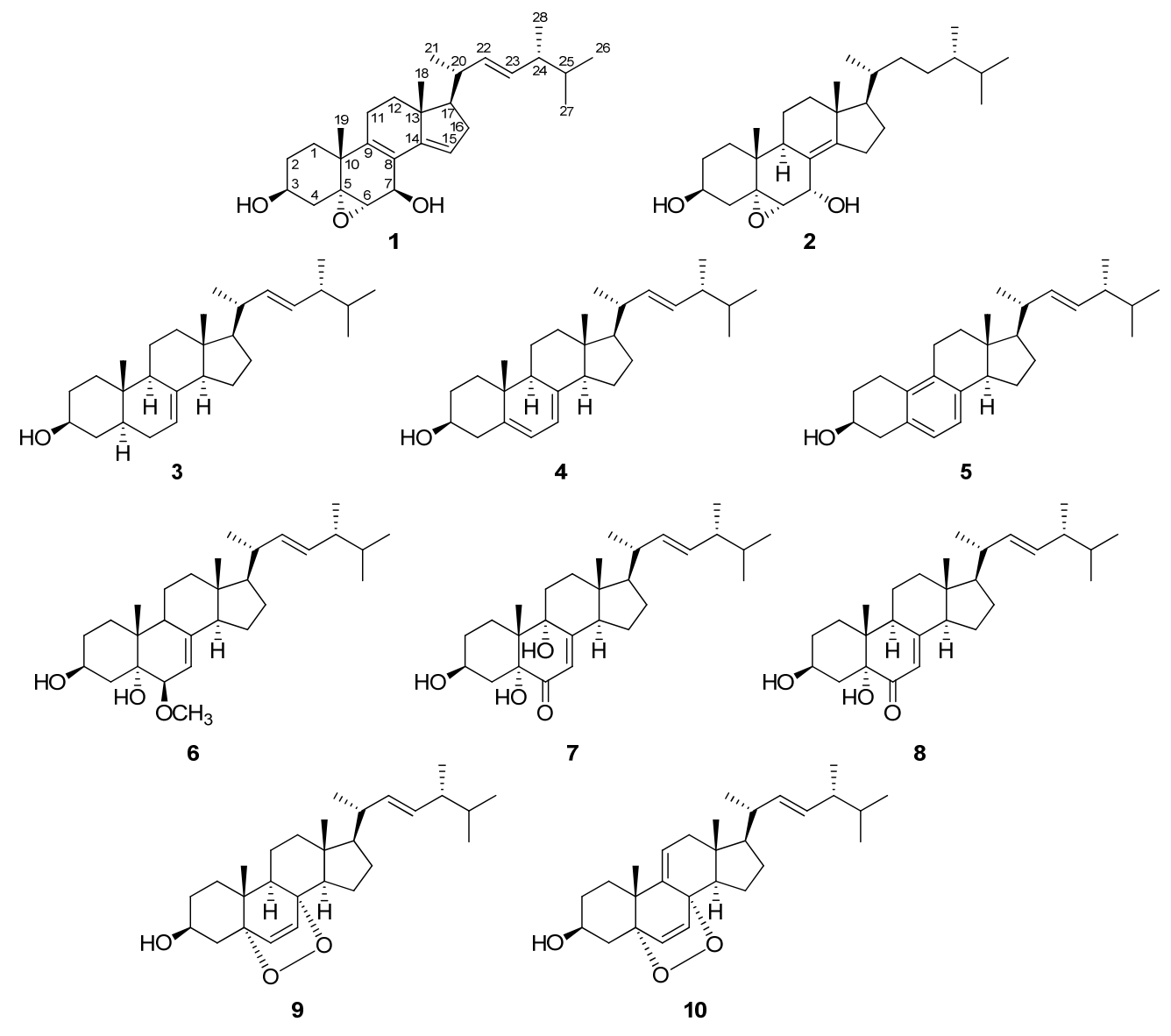

Figure 1. Structures of compounds.

Compound 1 was isolated as an amorphous solid, with a molecular formula of $\mathrm{C}_{28} \mathrm{H}_{42} \mathrm{O}_{3}$ by HREIMS. The infrared (IR) spectrum indicated the presence of hydroxy groups $\left(v_{\max } 3451 \mathrm{~cm}^{-1}\right)$, and the UV spectrum suggested the presence of a conjugated diene $\left(\lambda_{\max } 242.0 \mathrm{~nm}\right)$. The ${ }^{1} \mathrm{H}$ and ${ }^{13} \mathrm{C}$ NMR spectra $\left(\delta_{\mathrm{H}}\right.$ and $\delta_{\mathrm{C}}$ in ppm) in $\mathrm{CDCl}_{3}$ displayed signals for two tertiary methyls $\left(\delta_{\mathrm{H}} 0.82\right.$ (singlet $\left.\left.(\mathrm{s})\right), 1.30(\mathrm{~s})\right)$, four secondary methyls $\left(\delta_{\mathrm{H}} 0.83(\operatorname{doublet}(\mathrm{d})), 0.85(\mathrm{~d}), 0.93(\mathrm{~d}), 1.04(\mathrm{~d})\right)$, three oxymethines $\left(\delta_{\mathrm{H}} 3.24(\mathrm{~d})\right.$, 3.96 (triplet of triplets (tt)), 4.85 (broad singlet (br s)); $\delta_{\mathrm{C}} 59.5(\mathrm{~d}), 63.8(\mathrm{~d}), 68.4(\mathrm{~d})$ ), an sp ${ }^{3}$ oxygenated quaternary carbon $\left(\delta_{\mathrm{C}} 63.3(\mathrm{~s})\right)$, a tetrasubstituted olefin $\left(\delta_{\mathrm{C}} 122.2(\mathrm{~s}), 138.8(\mathrm{~s})\right)$, a trisubstituted olefin $\left(\delta_{\mathrm{H}} 5.55(\mathrm{br} \mathrm{s}) ; \delta_{\mathrm{C}} 118.7(\mathrm{~d}), 147.7(\mathrm{~s})\right)$, and a disubstutited olefin $\left(\delta_{\mathrm{H}} 5.20\right.$ (doublet of doublets $\left.(\mathrm{dd})\right)$, 
$\left.5.28(\mathrm{dd}) ; \delta_{\mathrm{C}} 132.4(\mathrm{~d}), 135.1(\mathrm{~d})\right)$ (Table 1, Figures S1-S4). In the HMBC spectrum, the correlations were observed as follows; Me-19 $\left(\delta_{\mathrm{H}} 0.82(\mathrm{~s})\right) / \mathrm{C}-5\left(\delta_{\mathrm{C}} 63.3(\mathrm{~s})\right), \mathrm{C}-9\left(\delta_{\mathrm{C}} 138.8(\mathrm{~s})\right) ; \mathrm{H}-7\left(\delta_{\mathrm{H}} 4.85(\mathrm{br} \mathrm{s})\right) / \mathrm{C}-5$ $\left(\delta_{\mathrm{C}} 63.3(\mathrm{~s})\right), \mathrm{C}-9\left(\delta_{\mathrm{C}} 138.8(\mathrm{~s})\right), \mathrm{C}-14\left(\delta_{\mathrm{C}} 147.7(\mathrm{~s})\right) ; \mathrm{H}-6\left(\delta_{\mathrm{H}} 3.24(\mathrm{~d})\right) / \mathrm{C}-7\left(\delta_{\mathrm{C}} 63.8(\mathrm{~d})\right), \mathrm{C}-8\left(\delta_{\mathrm{C}} 122.2(\mathrm{~s})\right)$; Me-18 $\left(\delta_{\mathrm{H}} 0.82(\mathrm{~s})\right) / \mathrm{C}-14\left(\delta_{\mathrm{C}} 147.7(\mathrm{~s})\right) ; \mathrm{Me}-28\left(\delta_{\mathrm{H}} 0.93(\mathrm{~d})\right) / \mathrm{C}-23$ and C-25 (Figure $2 \mathrm{~A}$ and Figure S5). The correlations between $\mathrm{H}_{2}-1-\mathrm{H}_{2}-2-\mathrm{H}-3\left(\delta_{\mathrm{H}} 3.96(\mathrm{tt})\right)-\mathrm{H}_{2}-4 ; \mathrm{H}-6\left(\delta_{\mathrm{H}} 3.24(\mathrm{~d})\right)-\mathrm{H}-7\left(\delta_{\mathrm{H}} 4.85(\mathrm{br} \mathrm{s})\right) ; \mathrm{H}-15$ $\left(\delta_{\mathrm{H}} 5.55(\mathrm{br} \mathrm{s})\right)-\mathrm{H}_{2}-16-\mathrm{H}-17-\mathrm{H}-20-\mathrm{Me}-21 ; \mathrm{H}-20-\mathrm{H}-22\left(\delta_{\mathrm{H}} 5.20(\mathrm{dd})\right)-\mathrm{H}-23\left(\delta_{\mathrm{H}} 5.28(\mathrm{dd})\right)-\mathrm{H}-24-\mathrm{Me}-28$ $\left(\delta_{\mathrm{H}} 0.93(\mathrm{~d})\right)$; Me-26 $\left(\delta_{\mathrm{H}} 0.85(\mathrm{~d})\right)-\mathrm{H}-25-\mathrm{Me}-27\left(\delta_{\mathrm{H}} 0.83(\mathrm{~d})\right)$ were observed in the ${ }^{1} \mathrm{H}^{1}{ }^{1} \mathrm{H}$ COSY spectrum (Figure 2A and Figure S6). From the above, the planar structure was determined as shown in Figure 2A. The configuration of the hydroxy groups at the $\mathrm{C}-3$ position was determined as $\beta$-orientation because of the coupling constant $(J)\left(\delta_{\mathrm{H}} 3.96(\mathrm{tt}, 11.5,5.4 \mathrm{~Hz})\right)$. The NOE correlation of Me-19/H-6ß (equatrial) suggested that the epoxy group at C-5,6 was $\alpha$-oriented, and that of $\mathrm{H}-7 \alpha / \mathrm{H}-15$ suggested that $7-\mathrm{OH}$ was $\beta$-oriented (Figure 2B and Figure S7). The geometry of the double bond at $\mathrm{C}-22$ was determined as $E$ from the coupling constants of $\mathrm{H}-22\left(\delta_{\mathrm{H}} 5.20(\mathrm{dd}, J=15.2,7.6 \mathrm{~Hz})\right)$ and $\mathrm{H}-23\left(\delta_{\mathrm{H}} 5.28(\mathrm{dd}, J=15.2\right.$, $7.9 \mathrm{~Hz})$ ). Comparison of ${ }^{13} \mathrm{C}$ NMR chemical shifts at C-24 $\left(\delta_{\mathrm{C}} 42.8\right)$ and $28\left(\delta_{\mathrm{C}} 17.6\right)$ with those of $24 R\left(\delta_{\mathrm{C}} 42.9(\mathrm{C}-24)\right.$ and $\left.17.7(\mathrm{C}-28)\right)$ and $24 S\left(\delta_{\mathrm{C}} 43.2(\mathrm{C}-24)\right.$ and $\left.18.1(\mathrm{C}-28)\right)$ methylcholestane-type sterols $[23,24]$ established the stereochemistry of $C-24$ as $R$. Therefore compound $\mathbf{1}$ was determined as (22E)-5 $\alpha, 6 \alpha$-epoxyergosta-8,14,22-triene-3 $3,7 \beta$-diol (Figure 1, Table S1). Compound 1 was similar to $(22 E)-5 \alpha, 6 \alpha$-epoxy-ergosta-8,14,22-triene-7 $\beta, 7 \alpha$-diol [25], except for the absence of a $7 \alpha$-hydroxy group and the presence of a $7 \beta$-hydroxy group. There are differences in $\delta_{\mathrm{H}}$ value measured with $\mathrm{C}_{6} \mathrm{D}_{6}$ such as $\mathrm{H}-7\left(7 \alpha\right.$-hydroxy-type: $\delta_{\mathrm{H}} 4.34(1 \mathrm{H}, \mathrm{dd}, J=11.2,2.6 \mathrm{~Hz})$ [25] vs. $7 \beta$-hydroxy-type (1): $\left.\delta_{\mathrm{H}} 4.74(\mathrm{br} \mathrm{s})\right)$, and H-15 (7 $\alpha$-hydroxy-type: $\delta_{\mathrm{H}} 6.50(1 \mathrm{H}, \mathrm{dd}, J=3.3,1.8 \mathrm{~Hz})$ [25] vs. $7 \beta$-hydroxy-type (1): $\delta_{\mathrm{H}} 5.33$ (br s)).

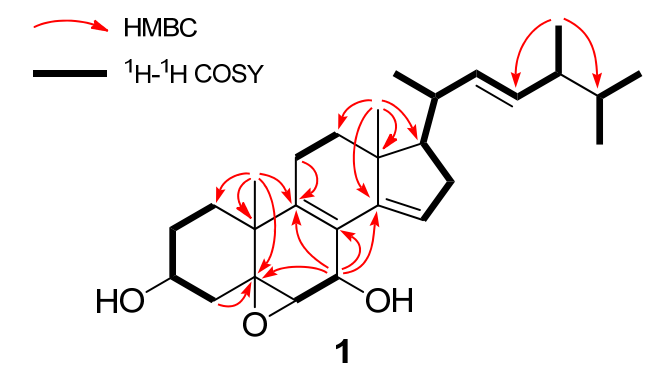

(A)

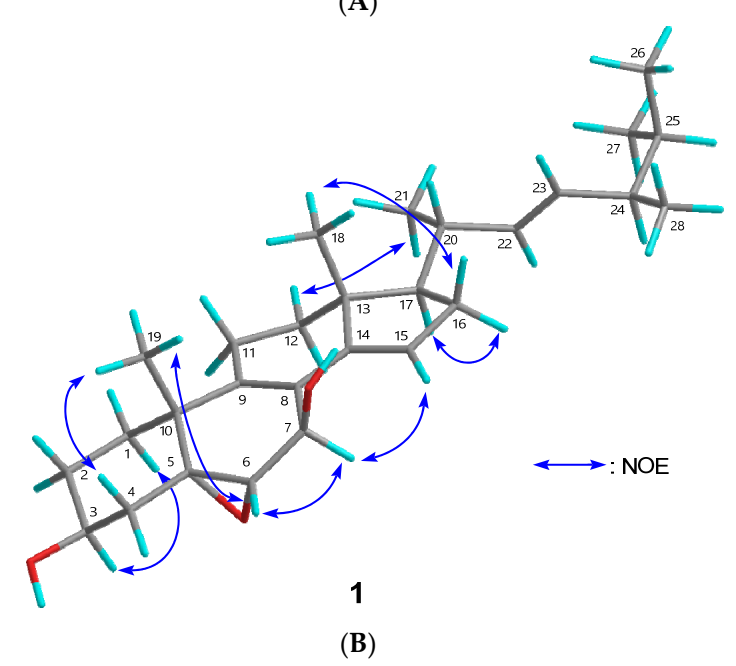

Figure 2. Structure determination of compound 1. (A) Key HMBC and ${ }^{1} \mathrm{H}_{-}{ }^{1} \mathrm{H} C O S Y$ correlations of compound 1; (B) Key NOE correlations of compound 1. The atoms of C, H, and O were shown in grey, aqua, and red, respectively. 
Table 1. ${ }^{1} \mathrm{H}$ and ${ }^{13} \mathrm{C}$ NMR Data for Compounds 1 and 2 in $\mathrm{CDCl}_{3}(\delta$ in ppm; $J$ in $\mathrm{Hz})$.

\begin{tabular}{|c|c|c|c|c|c|c|c|c|}
\hline \multirow{3}{*}{$\frac{\text { Position }}{1 \alpha}$} & \multicolumn{4}{|c|}{1} & \multicolumn{4}{|c|}{2} \\
\hline & \multicolumn{2}{|r|}{$\delta_{\mathbf{H}}$} & \multicolumn{2}{|c|}{$\delta_{\mathrm{C}}$} & \multicolumn{2}{|r|}{$\delta_{\mathbf{H}}$} & \multicolumn{2}{|c|}{$\delta_{\mathrm{C}}$} \\
\hline & 2.01 & $(1 \mathrm{H}$, multiplet $(\mathrm{m}))$ & 31.0 & $\mathrm{t}$ & 1.46 & $(1 \mathrm{H}, \mathrm{m})$ & 32.2 & $\mathrm{t}$ \\
\hline $1 \beta$ & 1.86 & $(1 \mathrm{H}, \mathrm{m})$ & & & 1.67 & $(1 \mathrm{H}, \mathrm{m})$ & & \\
\hline 2 & 1.68 & $(2 \mathrm{H}, \mathrm{m})$ & 30.9 & $\mathrm{t}$ & $\begin{array}{l}\alpha 1.96 \\
\beta 1.56\end{array}$ & $\begin{array}{l}(1 \mathrm{H}, \mathrm{m}) \\
(1 \mathrm{H}, \mathrm{m})\end{array}$ & 31.1 & $\mathrm{t}$ \\
\hline 3 & 3.96 & $(1 \mathrm{H}, \mathrm{tt}, J=11.5,5.4)$ & 68.4 & $\mathrm{~d}$ & 3.92 & $(1 \mathrm{H}, \mathrm{tt}, J=11.4,3.0)$ & 68.7 & d \\
\hline $4 \alpha$ & 1.50 & $(1 \mathrm{H}, \mathrm{m})$ & 39.0 & $\mathrm{t}$ & 1.42 & $(1 \mathrm{H}, \mathrm{m})$ & 39.6 & $\mathrm{t}$ \\
\hline $4 \beta$ & 2.21 & $(1 \mathrm{H}, \mathrm{m})$ & & & 2.13 & $\begin{array}{c}(1 \mathrm{H}, \mathrm{dd}, J=13.2 \\
11.4)\end{array}$ & & \\
\hline 5 & & & 63.3 & $\mathrm{~s}$ & & & 67.8 & $\mathrm{~s}$ \\
\hline 6 & 3.24 & $(1 \mathrm{H}, \mathrm{d}, J=2.4)$ & 59.5 & $\mathrm{~d}$ & 3.15 & $(1 \mathrm{H}, \mathrm{d}, J=3.5)$ & 61.3 & d \\
\hline 7 & 4.85 & $(1 \mathrm{H}, \mathrm{br} \mathrm{s})$ & 63.8 & $\mathrm{~d}$ & 4.43 & $(1 \mathrm{H}, \mathrm{dd}, J=9.6,3.5)$ & 65.1 & d \\
\hline 8 & & & 122.2 & $\mathrm{~s}$ & & & 125.1 & $\mathrm{~s}$ \\
\hline 9 & & & 138.8 & $\mathrm{~s}$ & 2.35 & $(1 \mathrm{H}, \mathrm{m})$ & 38.7 & d \\
\hline 10 & & & 38.3 & $\mathrm{~s}$ & & & 35.8 & $\mathrm{~s}$ \\
\hline 11 & 2.19 & $(2 \mathrm{H}, \mathrm{m})$ & 22.2 & $\mathrm{t}$ & $\begin{array}{l}\alpha 1.49 \\
\beta 1.40\end{array}$ & $\begin{array}{l}(1 \mathrm{H}, \mathrm{m}) \\
(1 \mathrm{H}, \mathrm{m})\end{array}$ & 19.0 & $\mathrm{t}$ \\
\hline $12 \alpha$ & 1.47 & $(1 \mathrm{H}, \mathrm{m})$ & 35.4 & $\mathrm{t}$ & 1.16 & $(1 \mathrm{H}, \mathrm{m})$ & 36.7 & $\mathrm{t}$ \\
\hline $12 \beta$ & 1.99 & $(1 \mathrm{H}, \mathrm{m})$ & & & 1.95 & $(1 \mathrm{H}, \mathrm{m})$ & & \\
\hline 13 & & & 44.6 & $\mathrm{~s}$ & & & 43.1 & $\mathrm{~s}$ \\
\hline 14 & & & 147.7 & s & & & 152.7 & $\mathrm{~s}$ \\
\hline 15 & 5.55 & $(1 \mathrm{H}, \mathrm{br} \mathrm{s})$ & 118.7 & d & $\begin{array}{l}\alpha 2.65 \\
\beta 2.30\end{array}$ & $\begin{array}{l}(1 \mathrm{H}, \mathrm{m}) \\
(1 \mathrm{H}, \mathrm{m})\end{array}$ & 25.0 & $\mathrm{t}$ \\
\hline $16 \alpha$ & 2.27 & $(1 \mathrm{H}, \mathrm{m})$ & & & 1.89 & $(1 \mathrm{H}, \mathrm{m})$ & 26.6 & $\mathrm{t}$ \\
\hline $16 \beta$ & 2.08 & $(1 \mathrm{H}, \mathrm{m})$ & 36.8 & $\mathrm{t}$ & 1.41 & $(1 \mathrm{H}, \mathrm{m})$ & & \\
\hline 17 & 1.55 & $(1 \mathrm{H}, \mathrm{m})$ & 56.4 & d & 1.21 & $(1 \mathrm{H}, \mathrm{m})$ & 56.6 & d \\
\hline 18 & 0.82 & $(3 \mathrm{H}, \mathrm{s})$ & 15.6 & quartet (q) & 0.85 & $(3 \mathrm{H}, \mathrm{s})$ & 17.9 & $\mathrm{q}$ \\
\hline 19 & 1.30 & $(3 \mathrm{H}, \mathrm{s})$ & 23.6 & $\mathrm{q}$ & 0.87 & $(3 \mathrm{H}, \mathrm{s})$ & 16.5 & $\mathrm{q}$ \\
\hline 20 & 2.24 & $(1 \mathrm{H}, \mathrm{m})$ & 38.8 & d & 1.46 & $(1 \mathrm{H}, \mathrm{m})$ & 34.9 & $\mathrm{~d}$ \\
\hline 21 & 1.04 & $(3 \mathrm{H}, \mathrm{d}, J=6.5)$ & 21.0 & $\mathrm{q}$ & 0.93 & $(3 \mathrm{H}, \mathrm{d}, J=6.8)$ & 19.1 & $q$ \\
\hline 22 & 5.20 & $(1 \mathrm{H}, \mathrm{dd}, J=15.2,7.6)$ & 135.1 & d & $\begin{array}{l}\text { A } 1.03 \\
\text { B } 1.44\end{array}$ & $\begin{array}{l}(1 \mathrm{H}, \mathrm{m}) \\
(1 \mathrm{H}, \mathrm{m})\end{array}$ & 33.4 & $\mathrm{t}$ \\
\hline 23 & 5.28 & $(1 \mathrm{H}, \mathrm{dd}, J=15.2,7.9)$ & 132.4 & d & $\begin{array}{l}\text { A } 0.95 \\
\text { B } 1.37\end{array}$ & $\begin{array}{l}(1 \mathrm{H}, \mathrm{m}) \\
(1 \mathrm{H}, \mathrm{m})\end{array}$ & 30.4 & $\mathrm{t}$ \\
\hline 24 & 1.88 & $(1 \mathrm{H}, \mathrm{m})$ & 42.8 & $\mathrm{~d}$ & 1.21 & $(1 \mathrm{H}, \mathrm{m})$ & 39.1 & d \\
\hline 25 & 1.48 & $(1 \mathrm{H}, \mathrm{m})$ & 33.1 & $\mathrm{~d}$ & 1.58 & $(1 \mathrm{H}, \mathrm{m})$ & 31.5 & $\mathrm{~d}$ \\
\hline 26 & 0.85 & $(3 \mathrm{H}, \mathrm{d}, J=6.8)$ & 19.9 & q & 0.85 & $(3 \mathrm{H}, \mathrm{d}, J=7.1)$ & 20.5 & $\mathrm{q}$ \\
\hline 27 & 0.83 & $(3 \mathrm{H}, \mathrm{d}, J=6.8)$ & 19.6 & $\mathrm{q}$ & 0.78 & $(3 \mathrm{H}, \mathrm{d}, J=7.0)$ & 17.6 & $\mathrm{q}$ \\
\hline 28 & 0.93 & $(3 \mathrm{H}, \mathrm{d}, J=6.8)$ & 17.6 & $\mathrm{q}$ & 0.77 & $(3 \mathrm{H}, \mathrm{d}, J=6.9)$ & 15.4 & $\mathrm{q}$ \\
\hline
\end{tabular}

Compound 2 was isolated as an amorphous solid, with a molecular formula of $\mathrm{C}_{28} \mathrm{H}_{46} \mathrm{O}_{3}$. The IR spectrum suggested the presence of hydroxy groups $\left(3387 \mathrm{~cm}^{-1}\right)$. The ${ }^{1} \mathrm{H},{ }^{13} \mathrm{C}$ NMR and HSQC spectra indicated the presence of two tertiary methyls $\left(\delta_{\mathrm{H}} 0.85(\mathrm{~s}), 0.87(\mathrm{~s})\right)$, four secondary methyls $\left(\delta_{\mathrm{H}} 0.77(\mathrm{~d})\right.$, $0.78(\mathrm{~d}), 0.85(\mathrm{~d}), 0.93(\mathrm{~d}))$, two oxymethines $\left(\delta_{\mathrm{H}} 3.92(\mathrm{tt}), 4.43(\mathrm{dd}) ; \delta_{\mathrm{C}} 65.1(\mathrm{~d}), 68.7(\mathrm{~d})\right)$, a trisubstituted $\operatorname{epoxy}\left(\delta_{\mathrm{H}} 3.15(\mathrm{~d}) ; \delta_{\mathrm{C}} 61.3(\mathrm{~d}), 67.8(\mathrm{~s})\right)$, and a tetrasubstituted olefin $\left(\delta_{\mathrm{C}} 125.1(\mathrm{~s}), 152.7(\mathrm{~s})\right)$ (Table 1 , Figures S8-S11). Based on the correlations at Me-18/C-14 ( $\left.\delta_{\mathrm{C}} 152.7(\mathrm{~s})\right), \mathrm{Me}-19 / \mathrm{C}-5\left(\delta_{\mathrm{C}} 67.8(\mathrm{~s})\right)$, and $\mathrm{H}-15 / \mathrm{C}-8\left(\delta_{\mathrm{C}} 125.1(\mathrm{~s})\right)$ and $\mathrm{C}-14\left(\delta_{\mathrm{C}} 152.7(\mathrm{~s})\right)$ in the HMBC spectrum, and $\mathrm{H}_{2}-1-\mathrm{H}_{2}-2-\mathrm{H}-3$ $\left(\delta_{\mathrm{H}} 3.92(\mathrm{tt})\right)-\mathrm{H}_{2}-4 ; \mathrm{H}-6\left(\delta_{\mathrm{H}} 3.15(\mathrm{~d})\right)-\mathrm{H}-7\left(\delta_{\mathrm{H}} 4.43(\mathrm{dd})\right)$ in the ${ }^{1} \mathrm{H}-{ }^{1} \mathrm{H}$ COSY spectrum (Figure $3 \mathrm{~A}$, Figures S12 and S13), oxymethines were at $\mathrm{C}-3$ and $\mathrm{C}-7$ positions, a trisubstituted epoxy group at the C-5, 6 positions, and a tetrasubstituted olefin at the C-8, 14 positions (Figure $3 \mathrm{~A}$ ). The NOE correlation between H-7 and Me-19 demonstrated the configuration of the hydroxy group at the C-7 position as $\alpha$-orientation (Figure 3B and Figure S14). The NOE correlation between H-4 $\beta$ and Me-19 suggested the orientation of the epoxy group at C-5, 6 was $\alpha$ (Figure 3B and Figure S14). The stereochemistry of C-24 was established as $S$ by comparison of the ${ }^{1} \mathrm{H}$ NMR chemical shift at Me-28 $\left(\delta_{\mathrm{H}} 0.77\right)$ with those of $24 R\left(\delta_{\mathrm{H}} 0.802\right)$ and $24 S\left(\delta_{\mathrm{H}} 0.781\right)$ ergostane-type sterols [26,27]. Therefore, the structure of 2 was established as $5 \alpha, 6 \alpha$-epoxyergosta- 8 (14)-ene- $3 \beta, 7 \alpha$-diol (Figure 1, Table S2). 


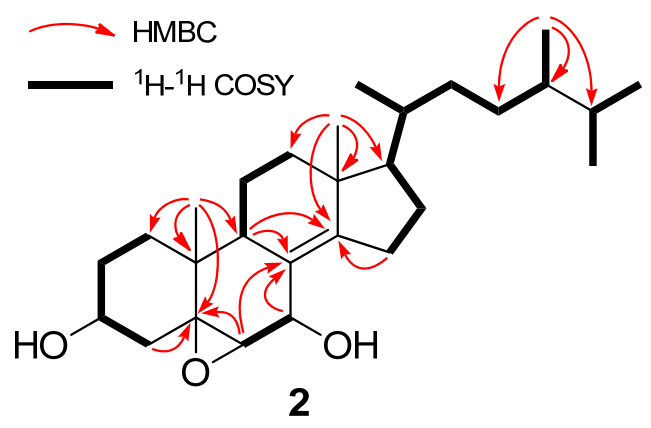

(A)

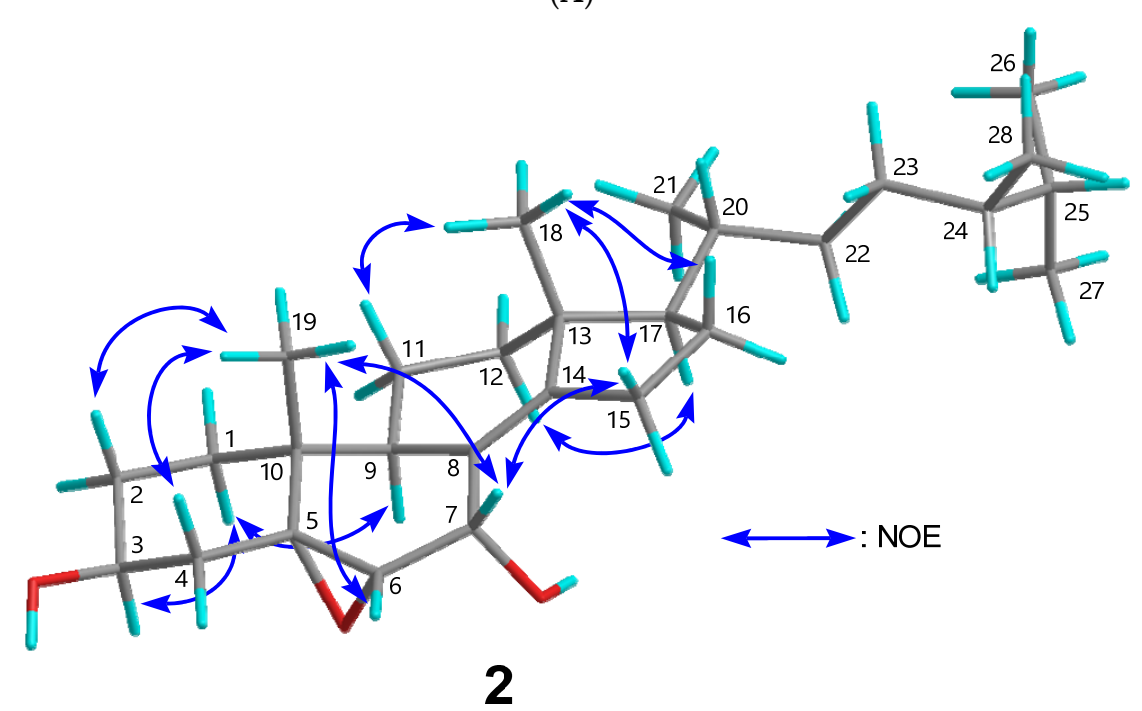

(B)

Figure 3. Structure determination of compound 2. (A) Key HMBC and ${ }^{1} \mathrm{H}_{-}{ }^{1} \mathrm{H}$ COSY correlations of compound 2; (B) Key NOE correlations of compound 2. The atoms of C, H, and $\mathrm{O}$ were shown in grey, aqua, and red, respectively.

\subsection{Evaluation for Aromatase Inhibitory Effects}

Compounds 1-10 and aminoglutethimide, a positive control, were evaluated for their aromatase inhibitory activities. Compounds 4 and 6 exhibited comparable inhibitory activities $\left(\mathrm{IC}_{50} 4: 8.1 \mu \mathrm{M}\right.$; 6: $2.8 \mu \mathrm{M}$ ) to aminoglutethimide ( $\mathrm{IC}_{50} 2.0 \mu \mathrm{M}$ ) (Figure $4 \mathrm{~A}$ ). Compounds 1, 3, 5, and $\mathbf{1 0}$ showed moderate activities ( $\mathrm{IC}_{50}$ 1: $17.3 \mu \mathrm{M} ; 3$ : $66.1 \mu \mathrm{M} ; 5$ : $33.8 \mu \mathrm{M} ; 10$ : $32.6 \mu \mathrm{M}$ ) (Figure $4 \mathrm{~B}$ ). Compounds 2, 7, 8, and 9 weakly inhibited aromatase (Figure 4B). Above results suggested that compounds 4 and 6 can be regarded as potential anti-breast cancer agents targeting aromatase. Based on the results in the figures, the following structure-activity relationship of the compounds can be concluded: (i) The double-bond at C-5, 6 intensifies the aromatase inhibitory activity in ergost-7-ene compounds ( $3\left(\mathrm{IC}_{50} 66.1 \mu \mathrm{M}\right) \mathrm{vs}$. $4\left(\mathrm{IC}_{50} 8.1 \mu \mathrm{M}\right)$ ); (ii) 9(11)-double-bond enhances the inhibitory activity in $5 \alpha, 8 \alpha$-epidioxyergost-6-ene compounds (9 $\left(\mathrm{IC}_{50}>100 \mu \mathrm{M}\right)$ vs. $10\left(\mathrm{IC}_{50} 32.6 \mu \mathrm{M}\right)$ ); (iii) 7-ene-6-one compounds did not show this activity $\left(7\right.$ and $\left.8\left(\mathrm{IC}_{50}>100 \mu \mathrm{M}\right)\right)$. 
(A)

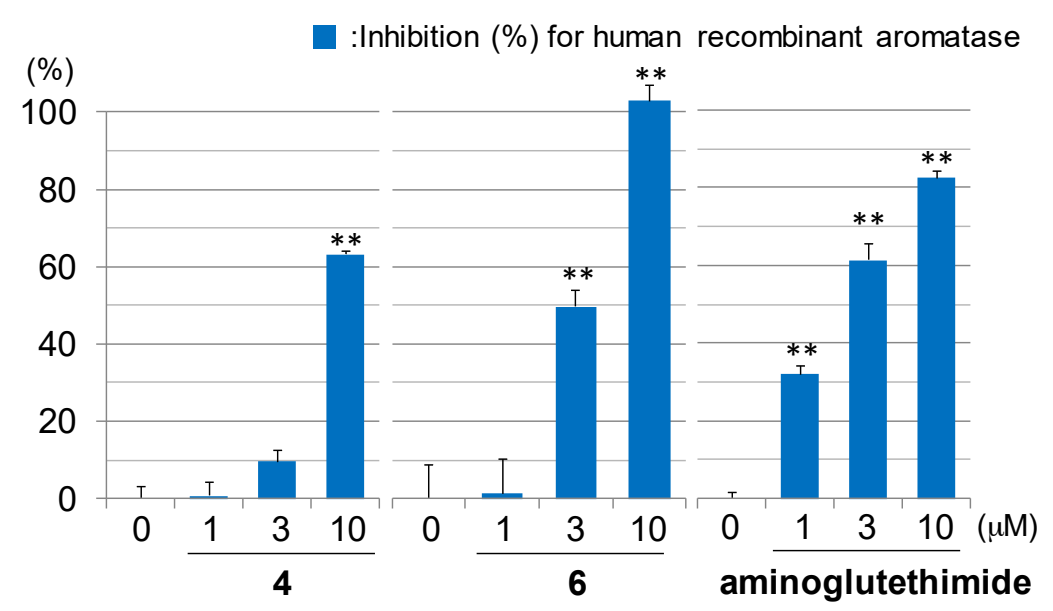

(B)
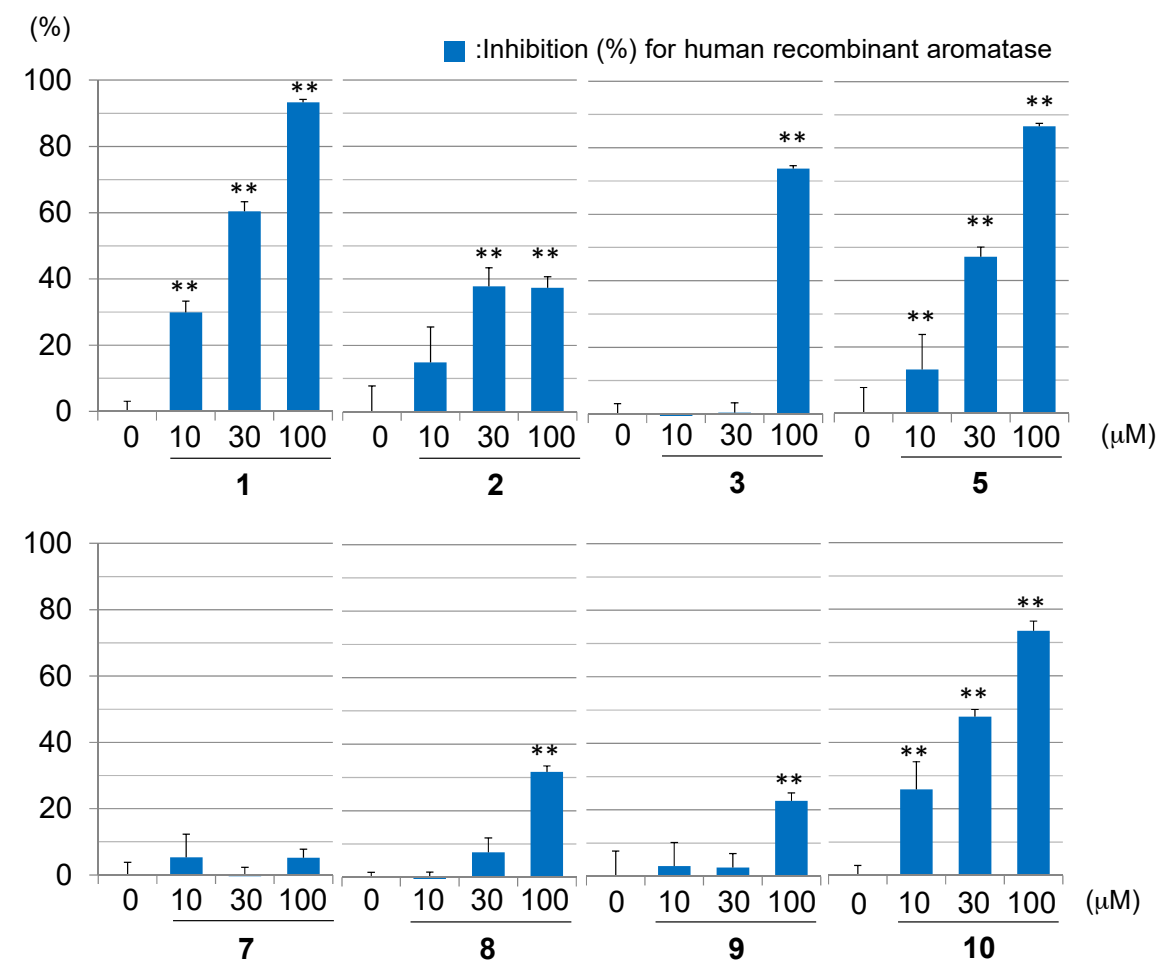

Figure 4. Inhibitory effects of sterols (1-10) from P. eryngii against human recombinant aromatase. (A) Inhibitory effects of sterols $(\mathbf{4}, \mathbf{6})$ and aminoglutethimide at 1, 3, and $10 \mu \mathrm{M}$. (B) Inhibitory effects of sterols (1-3, 5, 7-10) at 10, 30, and $100 \mu \mathrm{M}$. Each value represents the mean \pm the standard error (S.E.) of three determinations. Significant differences from the vehicle control $(0 \mu \mathrm{M})$ group shown as ** $p<0.01$.

\section{Experimental Section}

\subsection{General Methods}

Dibenzylfluorescein (DBF) and Human CYP19 + P450 Reductase SUPERSOMES (human recombinant aromatase) were obtained from BD Biosciences (Heidelberg, Germany). The physical data were obtained by the following instruments: a Yanagimoto micro-melting point apparatus for melting points (uncorrected); a JASCO DIP-1000 digital polarimeter for Optical rotations; a Perkin-Elmer 1720X FTIR spectrophotometer for IR spectra; an Agilent-NMR-vnmrs600 for the ${ }^{1} \mathrm{H}$ and ${ }^{13} \mathrm{C}$ NMR spectra $\left({ }^{1} \mathrm{H}: 600 \mathrm{MHz} ;{ }^{13} \mathrm{C}: 150 \mathrm{MHz}\right)$ in $\mathrm{CDCl}_{3}$ with tetramethylsilane as the internal standard; a Hitachi 
M-4000H double-focusing mass spectrometer for EIMS (70 eV). Column chromatography was carried out by Silica gel (70-230 mesh, Merck, Darmstadt, Germany) and silica gel 60 (230-400 mesh, Nacalai Tesque, Inc., Kyoto, Japan). HPLC was performed by the following systems; system I: Cosmosil 5SL-II column $\left(25 \mathrm{~cm} \times 20 \mathrm{~mm}\right.$ i.d.) (Nacalai Tesque, Inc.), hexane/EtOAc (5:1), $8.0 \mathrm{~mL} / \mathrm{min}, 35^{\circ} \mathrm{C}$; system II: Shimpack PREP-ODS $\left(25 \mathrm{~cm} \times 20 \mathrm{~mm}\right.$ i.d.) (Shimadzu corp., Kyoto, Japan), $\mathrm{MeOH}, 8.0 \mathrm{~mL} / \mathrm{min}, 35^{\circ} \mathrm{C}$; system III: Cosmosil $5 \mathrm{C}_{18}$-MS-II column ( $25 \mathrm{~cm} \times 20 \mathrm{~mm}$ i.d.) (Nacalai Tesque, Inc.), $\mathrm{MeOH} / \mathrm{H}_{2} \mathrm{O}$ (95:5), flow rate, $4.0 \mathrm{~mL} / \mathrm{min}, 35{ }^{\circ} \mathrm{C}$; system IV: Cosmosil $5 C_{18}-\mathrm{MS}-\mathrm{II}$ column, $\mathrm{MeOH} / \mathrm{H}_{2} \mathrm{O}$ (9:1), $4.0 \mathrm{~mL} / \mathrm{min}, 35^{\circ} \mathrm{C}$.

\subsection{Materials}

The fruiting bodies of P. eryngii were purchased from HOKUTO Corp. They were cultivated in Kagawa, Japan (Sample 1 in 2011, and Sample 2 in 2014). A voucher material has been deposited in the Herbarium of the Laboratory of Medicinal Chemistry, Osaka University of Pharmaceutical Sciences.

\subsection{Extraction and Isolation}

\subsubsection{Sample 1}

Sample 1 (fruiting bodies of P. eryngii (21 kg, fresh weight)) was extracted with $\mathrm{MeOH}$ under reflux ( 1 week, 4 times). The $\mathrm{MeOH}$ extract $\left(170 \mathrm{~g}\right.$ ) was then divided into EtOAc and $\mathrm{H}_{2} \mathrm{O}$ fractions by liquid-liquid partition. The EtOAc fraction $(60 \mathrm{~g})$ was separated into 20 fractions (Fr. S1-A to S1-T) with $\mathrm{SiO}_{2}$ column chromatography (CC) $\left(\mathrm{SiO}_{2}(3.5 \mathrm{~kg}) ; \mathrm{CHCl}_{3} / \mathrm{EtOAc}(1: 0\right.$ to 0:1), and EtOAc/MeOH (5:1, and 0:1)).

Fr. S1-H (836.5 mg), $\mathrm{CHCl}_{3} /$ EtOAc (10:1)-eluted fraction, was separated with $\mathrm{SiO}_{2} \mathrm{CC}$ to yield 8 fractions, S1-H1 to S1-H8. Preparative HPLC (system I) of S1-H3 (185.7 mg), hexane/EtOAc (5:1)-eluted fraction, provided 7 fractions, S1-H3-1 to SF3-7. S1-H3-4 was identified as 4 (31.8 mg; retention time $\left(t_{\mathrm{R}}\right) 19.2 \mathrm{~min}$ ). Preparative HPLC (system II) of $S 1-\mathrm{H} 3-5\left(5.4 \mathrm{mg}, t_{\mathrm{R}} 36.5 \mathrm{~min}\right.$ ) provided 3 (1.9 mg; $t_{\mathrm{R}} 37.5 \mathrm{~min}$ ). Preparative HPLC (system IV) of S1-H6 (14.3 mg), hexane/EtOAc (3:1)-eluted fraction, provided $10\left(1.3 \mathrm{mg}, t_{\mathrm{R}} 95.4 \mathrm{~min}\right)$ and $9\left(1.7 \mathrm{mg}, t_{\mathrm{R}} 120.2 \mathrm{~min}\right)$.

Fr. S1-I (1072.3 mg), $\mathrm{CHCl}_{3} / \mathrm{EtOAc}$ (10:1)-eluted fraction, was separated with $\mathrm{SiO}_{2} \mathrm{CC}$ to give 8 fractions, S1-I1 to S1-I10. Preparative HPLC (system I) of S1-I5 (45.2 mg), hexane/EtOAc (5:1)-eluted fraction, provided $5\left(2.8 \mathrm{mg}, t_{\mathrm{R}} 42.7 \mathrm{~min}\right)$.

\subsubsection{Sample 2}

Sample 2 (fruiting bodies of P. eryngii (120 kg, fresh weight)) was extracted with $\mathrm{MeOH}$ under reflux ( 3 days, 4 times). The $\mathrm{MeOH}$ extract $\left(2625 \mathrm{~g}\right.$ ) was divided into EtOAc and $\mathrm{H}_{2} \mathrm{O}$ fractions by liquid-liquid partition. The EtOAc fraction $(240 \mathrm{~g})$ was separated into 37 fractions (Fr. S2-A to S2-Z, and S2-a to S2-k) with $\mathrm{SiO}_{2}$ column chromatography (CC) $\left(\mathrm{SiO}_{2}(2.8 \mathrm{~kg}) ; \mathrm{CHCl}_{3} / \mathrm{EtOAc}(1: 0\right.$ to 0:1), and $\mathrm{MeOH})$.

Fr. S2-V (3964.9 mg), $\mathrm{CHCl}_{3} /$ EtOAc (1:1)-eluted fraction, was separated by $\mathrm{SiO}_{2} \mathrm{CC}$ to give 8 fractions, S2-V1 to S2-V21. Preparative HPLC (system III) of Fr. S2-V4 (110.9 mg), hexane/EtOAc (1:1)-eluted fraction, provided $8\left(1.5 \mathrm{mg} ; t_{\mathrm{R}} 36.9 \mathrm{~min}\right)$ and $\mathbf{6}\left(6.3 \mathrm{mg} ; t_{\mathrm{R}} 49.5 \mathrm{~min}\right)$. Preparative HPLC (system IV) of Fr. S2-V6 (451.6 mg), hexane/EtOAc (1:1)-eluted fraction, provided 7 (1.1 mg; $t_{\mathrm{R}} 59.8 \mathrm{~min}$ ). Preparative HPLC (system IV) of Fr. S2-V7 (270.5 mg), hexane/EtOAc (1:1)-eluted fraction, provided 2 ( $3.0 \mathrm{mg}$; $\left.t_{\mathrm{R}} 76.8 \mathrm{~min}\right)$. Preparative HPLC (system III) of Fr. S2-V10 (270.5 mg), hexane/EtOAc (1:1)-eluted fraction, provided $\mathbf{1}\left(1.9 \mathrm{mg} ; t_{\mathrm{R}} 36.3 \mathrm{~min}\right)$.

\subsection{3. (22E)- $5 \alpha, 6 \alpha$-Epoxyergosta-8,14,22-triene- $3 \beta, 7 \beta$-diol (1)}

$[\alpha]^{20}{ }_{\mathrm{D}}-23.6(c=0.13, \mathrm{EtOH}) ; \mathrm{IR} v_{\max }{ }^{\mathrm{KBr}} \mathrm{cm}^{-1}: 3451,2960,1697,1557,1456 ; \mathrm{UV} \lambda_{\max }{ }^{\mathrm{EtOH}} \mathrm{nm}(\log \varepsilon)$ :

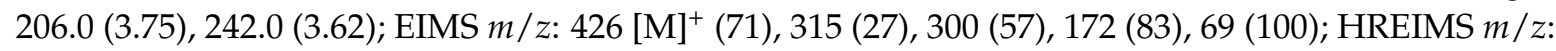
$426.3127[\mathrm{M}]^{+}$(calcd for 426.3134: $\left.\mathrm{C}_{28} \mathrm{H}_{42} \mathrm{O}_{3}\right) ;{ }^{1} \mathrm{H}$ NMR (400 MHz, $\left.\mathrm{C}_{6} \mathrm{D}_{6}\right) \delta_{\mathrm{H}}$ ppm: 0.80 (s, H-19), 
$0.91(\mathrm{~d}, 6.8 \mathrm{~Hz}), 0.93$ (d, $6.4 \mathrm{~Hz}), 1.01$ (d, $6.8 \mathrm{~Hz}), 1.07$ (d, $6.4 \mathrm{~Hz}), 1.12$ (s, H-18), 3.11 (d, $2.4 \mathrm{~Hz}, \mathrm{H}-6)$, 3.83 (tt, 11.6, 4.8 Hz, H-3), 4.74 (br s, H-7), 5.24 (dd, 15.6, 8.4 Hz, H-22), 5.32 (overlapped, H-23), 5.33 (br s, H-15).

\subsection{4. $5 \alpha, 6 \alpha$-Epoxyergost-8(14)-ene-3 $\beta, 7 \alpha$-diol (2)}

$[\alpha]^{20}{ }_{\mathrm{D}}-112.2(c=0.13, \mathrm{EtOH}) ; \mathrm{IR} v_{\max }{ }^{\mathrm{KBr}} \mathrm{cm}^{-1}: 3387,2959,2936,2871,1466,1377$; EIMS $\mathrm{m} / z$ : $430\left[^{[M]^{+}}\right.$(5), 412 (100), 394 (57), 379 (58), 267 (23), 213 (21); HREIMS m/z: 430.3450 [M] $^{+}$(calcd for 430.3447: $\left.\mathrm{C}_{28} \mathrm{H}_{46} \mathrm{O}_{3}\right)$.

\subsection{Inhibitory Effects against Human Recombinant Aromatase}

Inhibitory assay against human recombinant aromatase was performed as described previously $[28,29]$.

\subsection{Statistics}

Values are described as the mean \pm standard error of the mean (S.E.M.). Statistical analysis was performed by one-way analysis of variance, followed by Dunnett's test. Probability $(p)$ values less than 0.05 were regarded as significant.

\section{Conclusions}

In this study, we isolated two new sterols (1 and 2$)$ and elucidated their structures. They have $5 \alpha, 6 \alpha$-epoxy-7-hydroxy ergostane structure. In aromatase inhibitory assay, compounds 4 and 6 possessed comparable inhibitory effects $\left(\mathrm{IC}_{50} 4: 8.1 \mu \mathrm{M} ; 6: 2.8 \mu \mathrm{M}\right)$ against human recombinant aromatase to aminoglutethimide $\left(\mathrm{IC}_{50} 2.0 \mu \mathrm{M}\right)$. These results suggested that compounds 4 and 6 have potential as anti-breast cancer agents.

Supplementary Materials: Supplementary materials can be found at www.mdpi.com/1422-0067/18/11/2479/s1.

Acknowledgments: We thank Katsuhiko Minoura and Mihoyo Fujitake (Osaka University of Pharmaceutical Sciences) for the NMR and MS measurements.

Author Contributions: Takashi Kikuchi, Takeshi Yamada, Kiyofumi Ninomiya, Toshio Morikawa and Reiko Tanaka designed the experiments; Takashi Kikuchi, Naoki Motoyashiki, Takeshi Yamada and Reiko Tanaka isolated compounds, and elucidated their structures. Kanae Shibatani, Kiyofumi Ninomiya and Toshio Morikawa evaluated aromatase inhibitory effects of compounds. Takashi Kikuchi, Toshio Morikawa and Reiko Tanaka wrote the paper.

Conflicts of Interest: The authors declare no conflict of interest.

\section{Abbreviations}

NMR Nuclear magnetic resonance

HREIMS High resolution electron ionization mass spectrometry

$\mathrm{CDCl}_{3} \quad$ Duterated chloroform

HMBC Heteronuclear multiple bond coherence

COSY Correlation spectroscopy

NOE Nuclear overhauser effect

HSQC Hetero nuclear single quantum coherence

\section{References}

1. Hong, Y.; Li, H.; Yuan, Y.-C.; Chen, S. Molecular characterization of aromatase. Ann. N. Y. Acad. Sci. 2009, 1155, 112-120. [CrossRef] [PubMed]

2. Johnston, S.R.D.; Dowsett, M. Aromatase inhibitors for breast cancer: Lessons from the laboratory. Nat. Rev. Cancer 2003, 3, 821-831. [CrossRef] [PubMed]

3. Bae, K.; Yoo, H.-S.; Lamoury, G.; Boyle, F.; Rosenthal, D.S.; Oh, B. Acupuncture for aromatase inhibitor-induced arthralgia: A systematic review. Integr. Cancer Ther. 2015, 14, 496-502. [CrossRef] [PubMed] 
4. Balunas, M.J.; Kinghorn, A.D. Natural compounds with aromatase inhibitory activity: An update. Planta Med. 2010, 76, 1087-1093. [CrossRef] [PubMed]

5. Shimizu, K.; Yamanaka, M.; Gyokusen, M.; Kaneko, S.; Tsutsui, M.; Sato, J.; Sato, I.; Sato, M.; Kondo, R. Estrogen-like activity and prevention effect of bone loss in calcium deficient ovariectomized rats by the extract of Pleurotus eryngii. Phytother. Res. 2006, 20, 659-664. [CrossRef] [PubMed]

6. Rodriguez Estrada, A.E.; Royse, D.J. Yield, size and bacterial blotch resistance of Pleurotus eryngii grown on cottonseed hulls/oak sawdust supplemented with manganese, copper and whole ground soybean. Bioresour. Technol. 2007, 98, 1898-1906. [CrossRef] [PubMed]

7. Lee, I.-S.; Ryoo, I.-J.; Kwon, K.-Y.; Ahn, J.S.; Yoo, I.-D. Pleurone, a novel human neutrophil elastase inhibitor from the fruiting bodies of the mushroom Pleurotus eryngii var. ferulae. J. Antibiot. 2011, 64, 587-589. [CrossRef] [PubMed]

8. Kang, M.Y.; Rico, C.W.; Lee, S.C. In vitro antioxidative and antimutagenic activities of oak mushroom (Lentinus edodes) and king oyster mushroom (Pleurotus eryngii) byproducts. Food Sci. Biotechnol. 2012, 21, 167-173. [CrossRef]

9. Han, E.H.; Hwang, Y.P.; Kim, H.G.; Choi, J.H.; Im, J.H.; Yang, J.H.; Lee, H.-U.; Chun, S.-S.; Chung, Y.C.; Jeong, H.G. Inhibitory effect of Pleurotus eryngii extracts on the activities of allergic mediators in antigen-stimulated mast cells. Food Chem. Toxicol. 2011, 49, 1416-1425. [CrossRef] [PubMed]

10. Kawai, J.; Ouchi, K.; Inatomi, S.; Andoh, T. Pleurotus eryngii ameliorates lipopolysaccharide-induced lung inflammation in mice. Evid.-Based Complement. Altern. Med. 2014, 2014, 532389. [CrossRef] [PubMed]

11. Zhang, A.; Li, X.; Xing, C.; Yang, J.; Sun, P. Antioxidant activity of polysaccharide extracted from Pleurotus eryngii using response surface methodology. Int. J. Biol. Macromol. 2014, 65, 28-32. [CrossRef] [PubMed]

12. Yang, Z.; Xu, J.; Fu, Q.; Fu, X.; Shu, T.; Bi, Y.; Song, B. Antitumor activity of a polysaccharide from Pleurotus eryngii on mice bearing renal cancer. Carbohydr. Polym. 2013, 95, 615-620. [CrossRef] [PubMed]

13. Yaoita, Y.; Yoshihara, Y.; Kakuda, R.; Machida, K.; Kikuchi, M. New sterols from two edible mushrooms, Pleurotus eryngii and Panellus serotinus. Chem. Pharm. Bull. 2002, 50, 551-553. [CrossRef] [PubMed]

14. Wang, S.-J.; Li, Y.-X.; Bao, L.; Han, J.-J.; Yang, X.-L.; Li, H.-R.; Wang, Y.-Q.; Li, S.-J.; Liu, H.-W. Eryngiolide A, a cytotoxic macrocyclic diterpenoid with an unusual cyclododecane core skeleton produced by the edible mushroom Pleurotus eryngii. Org. Lett. 2012, 14, 3672-3675. [CrossRef] [PubMed]

15. Kikuchi, T.; Masumoto, Y.; In, Y.; Tomoo, K.; Yamada, T.; Tanaka, R. Eringiacetal A, 5,6-seco-(5S,6R,7R,9S)-5, 6:5,7:6,9-triepoxyergosta-8(14),22-diene-3 $\beta, 7 \beta$-diol, an unusual ergostane sterol from the fruiting bodies of Pleurotus eryngii. Eur. J. Org. Chem. 2015, 2015, 4645-4649. [CrossRef]

16. Kikuchi, T.; Maekawa, Y.; Tomio, A.; Masumoto, Y.; Yamamoto, T.; In, Y.; Yamada, T.; Tanaka, R. Six new ergostane-type steroids from king trumpet mushroom (Pleurotus eryngii) and their inhibitory effects on nitric oxide production. Steroids 2016, 115, 9-17. [CrossRef] [PubMed]

17. Keller, A.C.; Maillard, M.P.; Hostettmann, K. Antimicrobial steroids from the fungus Fomitopsis pinicola. Phytochemistry 1996, 41, 1041-1046. [CrossRef]

18. Seo Hyo, W.; Hung Tran, M.; Na, M.; Jung Hyun, J.; Kim Jin, C.; Choi Jae, S.; Kim Jung, H.; Lee, H.-K.; Lee, I.; Bae, K.; et al. Steroids and triterpenes from the fruit bodies of Ganoderma lucidum and their anti-complement activity. Arch. Pharm. Res. 2009, 32, 1573-1579. [CrossRef] [PubMed]

19. Barrero, A.F.; Oltra, J.E.; Poyatos, J.A.; Jimenez, D.; Oliver, E. Phycomysterols and Other Sterols from the Fungus Phycomyces blakesleeanus. J. Nat. Prod. 1998, 61, 1491-1496. [CrossRef] [PubMed]

20. Du, Z.-Z.; Shen, Y.-M. A rare new cleistanthane diterpene from the pericarp of Trewia nudiflora. Helv. Chim. Acta 2006, 89, 2841-2845. [CrossRef]

21. Kawagishi, H.; Katsumi, R.; Sazawa, T.; Mizuno, T.; Hagiwara, T.; Nakamura, T. Cytotoxic steroids from the mushroom Agaricus blazei. Phytochemistry 1988, 27, 2777-2779. [CrossRef]

22. Ishizuka, T.; Yaoita, Y.; Kikuchi, M. Sterol constituents from the fruit bodies of Grifola frondosa (Fr.) S.F. Gray. Chem. Pharm. Bull. 1997, 45, 1756-1760. [CrossRef]

23. Yan, X.-H.; Liu, H.-L.; Huang, H.; Li, X.-B.; Guo, Y.-W. Steroids with Aromatic A-Rings from the Hainan Soft Coral Dendronephthya studeri Ridley. J. Nat. Prod. 2011, 74, 175-180. [CrossRef] [PubMed]

24. Li, W.; Zhou, W.; Song, S.B.; Shim, S.H.; Kim, Y.H. Sterol Fatty Acid Esters from the Mushroom Hericium erinaceum and Their PPAR Transactivational Effects. J. Nat. Prod. 2014, 77, 2611-2618. [CrossRef] [PubMed] 
25. Ohnuma, N.; Amemiya, K.; Kakuda, R.; Yaoita, Y.; Machida, K.; Kikuchi, M. Sterol constituents from two edible mushrooms, Lentinula edodes and Tricholoma matsutake. Chem. Pharm. Bull. 2000, 48, 749-751. [CrossRef] [PubMed]

26. Rubinstein, I.; Goad, L.J.; Clague, A.D.H.; Mulheirn, L.J. The $220 \mathrm{MHz}$ NMR spectra of phytosterols. Phytochemistry 1976, 15, 195-200. [CrossRef]

27. Kobayashi, M.; Krishna, M.M.; Haribabu, B.; Anjaneyulu, V. Marine sterols. XXV. Isolation of 23-demethylgorgost-7-ene-3 $\beta, 5 \alpha, 6 \beta$-triol and (24S)-ergostane-3 $\beta, 5 \alpha, 6 \beta, 7 \beta, 15 \beta$-pentol from soft corals of the Andaman and Nicobar coasts. Chem. Pharm. Bull. 1993, 41, 87-89. [CrossRef]

28. Ninomiya, K.; Shibatani, K.; Sueyoshi, M.; Chaipech, S.; Pongpiriyadacha, Y.; Hayakawa, T.; Muraoka, O.; Morikawa, T. Aromatase Inhibitory Activity of Geranylated Coumarins, Mammeasins C and D, Isolated from the Flowers of Mammea siamensis. Chem. Pharm. Bull. 2016, 64, 880-885. [CrossRef] [PubMed]

29. Tanabe, G.; Tsutsui, N.; Shibatani, K.; Marumoto, S.; Ishikawa, F.; Ninomiya, K.; Muraoka, O.; Morikawa, T. Total syntheses of the aromatase inhibitors, mammeasins C and D, from Thai medicinal plant Mammea siamensis. Tetrahedron 2017, 73, 4481-4486. [CrossRef]

(C) 2017 by the authors. Licensee MDPI, Basel, Switzerland. This article is an open access article distributed under the terms and conditions of the Creative Commons Attribution (CC BY) license (http:/ / creativecommons.org/licenses/by/4.0/). 